Topics

\title{
Exploration of the Jovian System by EJSM (Europa Jupiter System Mission): Origin of Jupiter and Evolution of Satellites
}

\author{
By Sho SASAKI ${ }^{1)}$, Masaki FuJimoto ${ }^{2)}$, Takeshi TAKASHIMA ${ }^{2)}$, Hajime YANO ${ }^{2)}$, Yasumasa KASABA ${ }^{3)}$, \\ Yukihiro TAKAHASHI ${ }^{4}$, Jun KIMURA ${ }^{4)}$, Tatsuaki OKADA ${ }^{2)}$, Yasuhiro KAWAKATSU ${ }^{22}$, \\ Yuichi TSUdA ${ }^{2)}$, Jun-ichiro KAWAGUCHI ${ }^{2)}$, Ryu FUnASE ${ }^{2)}$, Osamu MORI ${ }^{2)}$, Mutsuko MORIMOTO ${ }^{2)}$, \\ Masahiro IKOMA ${ }^{5)}$, Takeshi NAGANUMA ${ }^{6}$, Atsushi YAMAJI ${ }^{7)}$, \\ Hauke HUSSMANN $^{8)}$, Kei KURITA ${ }^{9)}$ and JUPITER WORKING GROUP

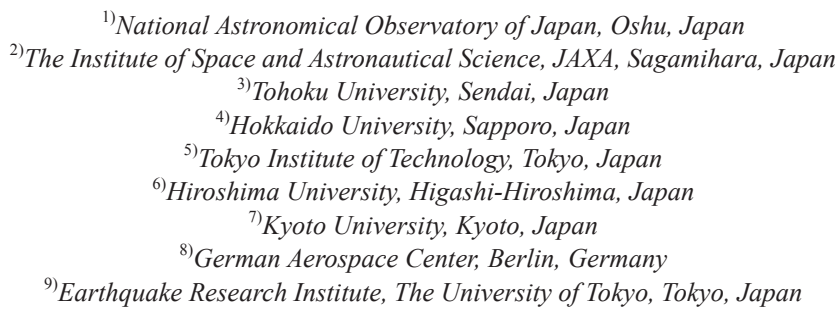

(Received July 16th, 2009)

\begin{abstract}
EJSM (Europa Jupiter System Mission) is a planned Jovian system mission with three spacecraft aiming at coordinated observations of the Jovian satellites especially Europa and the magnetosphere, atmosphere and interior of Jupiter. It was formerly called "Laplace" mission. In October 2007, it was selected as one of future ESA scientific missions Cosmic Vision (2015-2025). From the beginning, Japanese group is participating in the discussion process of the mission. JAXA will take a role on the magnetosphere spinner JMO (Jupiter Magnetosphere Orbiter). On the other hand, ESA will take charge of JGO (Jupiter Ganymede Orbiter) and NASA will be responsible for JEO (Jupiter Europa Orbiter). In February 2009, EJSM is prioritized as the first candidate of outer planet flagship mission and mission study continues in the course of Cosmic Vision. The expected launch time of EJSM will be expected in 2020. Currently we are seeking a possibility to combine JMO with a proposed solar sail mission of JAXA for Jupiter and one of Trojan asteroids.
\end{abstract}

Key Words: Jupiter, Europa, Ganymede, Jovian Magnetosphere, Trojan Asteroids

\section{Introduction: From Laplace to EJSM}

Jupiter is the largest and most massive planet in our solar system. It is a rapidly rotating gaseous body with main composition is hydrogen and helium. Recent discoveries of extrasolar planets have convinced researchers that Jupiter is the most prominent representative body not only in the solar system but also in the universe ${ }^{1)}$. Jupiter has more than 60 satellites, four of which (Io, Europa, Ganymede, and Callisto) were discovered by Galileo in 400 years ago. Jupiter has the strongest planetary magnetosphere in the solar system ${ }^{2}$.

The Jovian system was observed by Voyager 1 and 2, Cassini, and New Horizons by flybys, and was observed by Galileo orbiter and its atmospheric probe. However, very limited telemetry of Galileo spacecraft prohibited detailed data acquisition. Detailed satellite mapping should be done by satellite orbiters. Moreover, 3D structure of magnetosphere is revealed only by multi-spacecraft mission ${ }^{3)}$.

EJSM (Europa Jupiter System Mission) is a planned Jovian system mission with three spacecraft aiming at coordinated observations of the Jovian satellites especially Europa and the magnetosphere, atmosphere and interior of Jupiter ${ }^{3)}$. It was formerly called "Laplace" mission. From the beginning, JAXA and Japanese members committed with the mission planning. Initially it was ESA-JAXA mission where ESA would be responsible for a Jupiter-satellite orbiter and a Europa orbiter. JAXA would be responsible for a magnetospheric orbiter (JMO). Similar to ESA-JAXA Mercury mission BepiColombo, the initial plan was that JMO would be launched and transported together with one of ESA's orbiters. JAXA had also its original orbital study for Jupiter mission that will be launched by H-IIA vehicle ${ }^{4)}$. In October 2007, "Laplace" was selected as one of ESA's L-Class Cosmic Vision mission candidates (2015-2025) ${ }^{3)}$. Then NASA with a Europa orbiter participated in the mission plan. From 2008, ESA takes charge of JGO (Jupiter Ganymede Orbiter) and NASA is responsible for JEO (Jupiter Europa Orbiter). JAXA tekas a role on the JMO (Jupiter Magnetosphere Orbiter) as was before. A Europa lander is also studied by Russian Space Agency. In February 2009, NASA and ESA decided to continue the study of EJSM for a strong candidate of the outer solar system mission.

Launches of EJSM spacecraft will be expected in 2020 (or early 2020's). In the initial plan of EJSM, JMO would be launched together with ESA's JGO. However, from the resource and mass excess of JGO, JAXA is requested to 
launch JMO by its vehicle. Currently we are seeking a possibility to combine JMO with the formerly proposed solar sail mission of JAXA for Jupiter and one of Trojan asteroids.

\section{Mission with Solar Power Sail Project}

JAXA already started a study of a solar power sail for deep space explorations in early 2000's. The ISAS (Institute of Space and Astronautical Science) once evaluated a mission proposal for engineering verification spacecraft, which is called the solar power sail, a hybrid propulsion system of a solar sail and ion engines. The principle purpose is to demonstrate technologies necessary to explore the outer planet region. Together with a solar sail (photon propulsion), it should have very efficient ion engines where electric power is produced by very thin solar panels within the sail. JAXA has already experienced ion engines in the successful Hayabusa asteroid mission ${ }^{5)}$. And the engineering solar sail mission IKAROS (Interplanetary Kite-craft Accelerated by Radiation Of the Sun) will be launched in 2010."

Using a larger (100m-scale) solar power sail and efficient ion engines, the planned spacecraft can transfer large payload mass to Jovian system. According to a nominal mission plan, it will take about 4-6 years for spacecraft to go to Jupiter, and by the extended mission the spacecraft will arrive at one of Jovian $\mathrm{L}_{4}$ Trojan asteroids after using gravitational swing-by with Jupiter ${ }^{6}$. Currently we are studying a mission to Jupiter and one (or two) of Trojan asteroids, which are primitive bodies with information of the early solar system as well as raw solid materials of Jovian system. As the main spacecraft flies by Jupiter to direct Trojan asteroid using gravity assist, JMO will be released from the main solar sail spacecraft and inserted to the orbit around Jupiter using chemical thrusters. Apojove of JMO around Jupiter will be decreased by achemical thrusters and gravity assists by Galilean satellites.

\section{Science on the Jovian Origin and Satellites}

\subsection{Jupiter system}

The Jupiter System, with Jupiter and its satellites, can be considered as a small solar system ${ }^{7)}$. Detailed observation of Galilean satellites (Io, Europa, Ganymede, and Callisto) as well as smaller satellites such as Amalthea would be important for the study of the origin of Jovian system. Galilean satellites are considered to have accreted within the circumplanetary

\footnotetext{
* Additional note to the proof: IKAROS (Interplanetary Kite-craft Accelerated by Radiation Of the Sun) was launched successfully in May, 2011 together with Venus orbiter, AKATSUKI. Several engineering operations such as sail deployment, use of the radiation pressure, and power generation by thin-film solar panels, were fulfilled successfully. Two onboard science instrument (a dust detector and gamma ray a burst monitor) are being operated successfully.
}

gas disk (subnebula) around Jupiter, although some of Jovian satellites should have been captured later ${ }^{8)}$.

Among the moons, Europa is believed to have the interior ocean between the icy crust and silicate mantle ${ }^{8)}$. The crust of Europa would be tectonically active where evidence of erupted water with salts is observed on the surface. There would be carbonate-hydrate and/or sulfate-hydrate ${ }^{9)}$. The presence of the salty interior ocean raised interest in astrobiology; the occurrence of life in the Europan ocean is discussed ${ }^{10)}$. Ganymede is also considered to have the interior ocean between ice layers ${ }^{8)}$. Resurfacing processes on Europa and Ganymede should be studied to know the properties of the internal oceans. Not only the thickness of icy crust of Europa but also ocean thickness and its bottom topography should be important target. Compositions of the satellites of Jovian system in contrast to those of Saturnian and Uranian systems should provide a key for the origin of gas-rich planets.

Galileo spacecraft found that Ganymede has an intrinsic magnetic field, which would be operated by self-excited dynamo in the molten metallic core ${ }^{11)}$. Further observations are necessary to constrain the satellite magnetic field. The presence of active dynamo depends critically on the thermal state and internal structure. However, inferred interior structure using current gravity data has large uncertainty ${ }^{12)}$, and then the possibility of dynamo activity is unclear. The heat capacity that should control the cooling history of the core depends largely on core size and composition ${ }^{13)}$.

\subsection{Trojan asteroids}

Trojan asteroids, in other words Jupiter Trojans, are a large group of small bodies that share the orbit of the Jupiter around the Sun. Relative to Jupiter, each Trojan asteroid move around one of Jupiter's two Lagrangian points $\mathrm{L}_{4}$ and $\mathrm{L}_{5}$, which are $60^{\circ}$ ahead of and behind Jupiter in its orbit, respectively. Trojan asteroids are distributed in two elongated, curved regions along Jovian orbit around $\mathrm{L}_{4}$ or $\mathrm{L}_{5}$.

At present, there are two models for the origin of Trojan asteroids ${ }^{13)}$. One is that they are leftovers of the planetesimals that formed the Jovian systems. Another is so-called Nice Model: Trojan asteroids are captured when Jupiter and Saturn entered 1:2 resonance orbits ${ }^{13)}$. They should have come from outer solar system, probably some of Kuiper Belt objects.

Most of Jovian $\mathrm{L}_{4}$ Trojan asteroids are classified into D-type asteroids ${ }^{14)}$, poorly understood taxonomic types from neither ground observation spectroscopy nor meteoritic analyses due to the lack of possible analog chondrites discovered on the earth. One hypothesis is that D-type asteroids would be remnants of short-period cometary nucleus. If detailed observation revealed the connection between Trojan asteroids and cometary nucleus, this would support the Kuiper belt origin model of Trojan asteroids.

From the heritage of Hayabusa and Hayabusa-2 missions, we propose basic instrument packages consisting of a multiband UV-Visible high resolution imager (for geology and compositions), an infrared spectrometer with wavelength up to 3 micron (for composition and water/ice detection), and a 
laser altimeter (for geology, shape, and geodesy). A thermal IR sensor (for regolith properties) is optional. Different from Hayabusa and Hayabusa 2, we expect continuous coverage of spectra from 1 to 3 micron (using both spectrometers NIRS-1 and 2 on board Hayabusa and Hayabusa-2). This is important for ice/organic matter detection for the discussion of the origin of Trojan asteroids. Instruments for cruising science such as interplanetary dust, gamma ray burst and cosmic background are also proposed as discussed in Sec. 5.

\section{JMO Science on Jovian Magnetosphere}

Where the Jovian magnetosphere stands in the space plasma science is very clear. It is the gateway to the astrophysical plasma world. Explosive phenomena require ideal-MHD to break down. How this can be done in an astrophysical plasma situation where the basic scale length is much larger than the non-MHD characteristic scales (ion and electron scales) is not clear at all. Clarifying this certainly needs in-situ plasma measurements in an astro-like situation, and the huge Jovian magnetosphere is the right place. The Jovian magnetosphere is driven by the very fast rotation of Jupiter and populated by ions coming mainly from its satellites, especially Io. The regarding astrophysical processes are (i) dynamics of a magnetodisk with different mechanisms of angular momentum exchange and dissipation of rotational energy, (ii) electro-dynamical coupling between a central magnetic body and its satellites and, (iii) global and continuous acceleration of particles. As shown in Fig. 1, electrodynamic interactions of the Jovian system include generation of plasma at the Io torus, magnetosphere / satellites interactions, dynamics of a giant plasma disc coupled to Jupiter's rotation by the auroral current system, generation of Jupiter's intense radiation belts.

The Jovian magnetosphere is the gateway to the astro-world also in the sense that it is the most intense particle accelerator in the planetary system. Sulfur ions that came out of Io at the energy of less than $1 \mathrm{eV}$ are somehow energized up to $>10$ $\mathrm{MeV}$ within the magnetosphere. It is obvious that both turbulence driven by the fast rotation of the planet and magnetic reconnection in the magnetodisk are playing crucial roles but nailing down of the mechanisms is yet to be done.

Ganymede has an intrinsic magnetic field and occupies a small magnetosphere in the huge Jovian magnetosphere ${ }^{11)}$. Interaction between Jovian particles and Ganymedean magnetosphere is another intriguing target of plasma science. It is desirable that JMO shall monitor Jovian magnetosphere while JGO is observing Ganymedean environment. During the initial stage of orbital maneuver, JMO by itself will flyby with Ganymede and observe its environment.

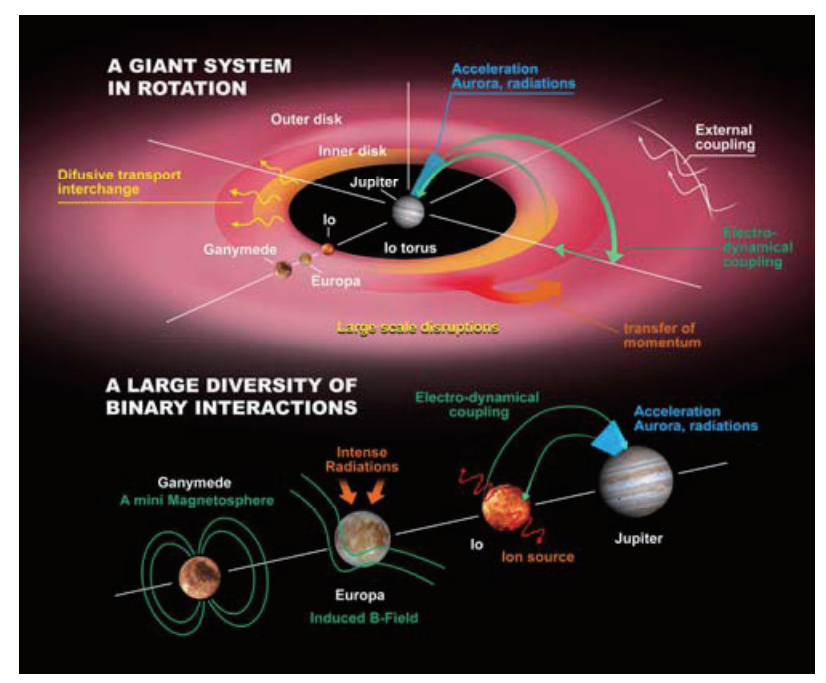

Fig. 1. Electrodynamic interactions in the Jovian environment. Plasma production at the Io torus, magnetosphere / satellites interactions, dynamics of a giant plasma disk coupled to Jupiter's rotation by the auroral current system, generation of Jupiter's intense radiation belts ${ }^{3}$.

The Japanese solar power sail mission with JMO will take about 6 years to Jupiter and 5 years more to one of Trojan asteroids around $\mathrm{L}_{4}$. Since the synodic encounter period between the Earth and Jupiter is about 400 days, we have the launch window nearly every year. In the baseline plan, JGO and JEO will be launched in 2020, if JGO is finally selected as the first L-class mission of Cosmic Vision. Since the cruising time of JMO is nearly the same as JEO and JGO, JMO can observe Jovian magnetosphere cooperatively from the beginning if we can launch JMO in 2020. Since the stages of orbiting around Jupiter are more than 2 years for JGO and JEO, even if JMO is launched in 2022, we can still execute cooperative observation with JGO and JEO when they are orbiting around Ganymede and Europa. The cruise time from the launch to Jupiter could be as short as 4 years. Then we can conduct nearly full cooperative observation from the beginning even if JMO is launched in 2022.

Collaborating with plasma instruments on board JEO and JGO, JMO will investigate the fast and huge rotating magnetosphere to clarify the energy procurement from Jovian rotation to the magnetosphere, to clarify the interaction between the solar wind and the magnetosphere. JMO will clarify the characteristics of the strongest accelerator in the solar system. JMO will investigate the role of Io as a source of heavy ions in the magnetosphere.

In the initial period, the Jovian magnetosphere is observed by three spacecraft (JMO, JGO, and JEO). JMO will take the largest apojove $\left(>100 \mathrm{R}_{\mathrm{J}}: \mathrm{R}_{\mathrm{J}}\right.$ being the radius of Jupiter, $71492 \mathrm{~km}$ ). If possible, we set perijove close to Io's orbit to observe the role of particles from Io on the magnetosphere. After JEO and JGO enter orbits around Europa and Ganymede, respectively, JMO takes equatorial orbits with perijove at Europa or Ganymede's orbit and apojove around $100 \mathrm{R}_{\mathrm{J}}$. JMO will determine the outer boundary condition 
when JEO and JGO observe the satellite-magnetosphere interaction. In the final stage after JEO and JGO stopped operation, using satellite flybys, orbital inclination of JMO can be increased to observe off-equatorial characteristic of magnetosphere and polar zones of Jupiter.

JMO will have an energetic particle instrument package covering wide range of energy. The package includes low-energy plasma spectrometers $(<$ a few $\mathrm{keV})$, medium energy particle detectors $(1-80 \mathrm{keV})$, energetic particle detectors $(50 \mathrm{keV}-1 \mathrm{MeV})$, very high energetic particle detectors $(>1 \mathrm{MeV})$ and DC electric field / magnetic field. JMO should have a plasma wave instrument package including a magnetometer and plasma/wave instrument. JMO will also have remote sensing instruments such as an ENA imager, an EUV spectrometer, and a camera for the observation of Jupiter, Io torus, and magnetosphere. JMO will have a dust detector (the impact-ionization type different from one in the main spacecraft) to investigate interaction between motion of fine dust particles and strong magnetic field. Both JGO and JEO will also have plasma instrument packages with a magnetometer ${ }^{3)}$.

\section{Other Science Topics}

The proposed mission will have long (more than 10 years) cruising time, where additional important science targets are discussed. The solar powered sail mission will have several opportunities to make flyby observations of main belt asteroids during the orbit to Jupiter. Studying zodiacal light reduction as a function of the heliocentric distance and the first observation of comic background radiation by dust-free infrared astronomy are intriguing targets. Both can be observed by the same photometer to produce data during the cruising, especially around 5AU. About $0.5 \%$ of the sail film may be replaced with PVDF (Polyvinylidene Fluoride) film threshold dust impact detectors ${ }^{12)}$, which the exposed area is two orders of magnitude larger than previous large dust detectors. A gamma-ray burst detector is also a candidate instrument. An interplanetary detector network for positioning of gamma-ray bursts in higher precision also becomes more advantageous as the spacecraft goes further out the earth. Both PVDF dust detector and a gamma-ray burst detector are on board IKAROS sail mission.

\section{Conclusion}

EJSM (Europa Jupiter System Mission) is a planned Jovian system mission with three spacecraft aiming at coordinated observations of the Jovian satellites especially coordinated observations of the Jovian satellites especially Jovian system mission with three spacecraft aiming at the magnetosphere spinner JMO. ESA will take charge of JGO and NASA will make JEO. Cooperative observation of Jovian magnetosphere by multi-spacecraft is one of the most important subjects in EJSM. We have been developing a scenario that JMO will be released and inserted to the orbit around Jupiter as solar power sail spacecraft flies by Jupiter finally aiming at one (or two) of Trojan asteroids.

\section{Acknowledgments}

Jupiter WG and Jupiter-Trojan WT are supported by ISAS/JAXA and JSPEC/JAXA.

\section{References}

1)Seager, S.: Exoplanet, Univ. Arizona Press, 2010.

2)Dessler, A. J.: Physics of the Jovian Magnetosphere. Cambridge University Press, New York, 1983.

3)Blanc, M. et al.: LAPLACE: A Mission to Europa and the Jupiter System for ESA's Cosmic Vision Programme, Experimental Astronomy, 23 (2009), pp.849-892.

4)Tsuda, Y.: Preliminary Orbit Design of Jupiter System Tour for Jovian Magnetospheric Science Mission, Proceedings of 17th Workshop on JAXA Astrodynamics and Flight Mechanics (2008), pp.304-310.

5)Kuninaka, H, Nishiyama, K., Funaki, I., Shimizu, Y., Yamada, T. and Kawaguchi, J.: Assessments of Plasma Interactions and Flight Status on HAYABUSA Asteroid Explorer Propelled by Microwave Discharge Ion Engines, IEEE Transaction on Plasma Science, 34 (2006), pp.2125-2132

6)Yano, H., et al.: Scientific Observations onboard the JAXA/ISAS Solar Powered Sail Mission, 35th COSPAR Scientific Assembly. Held 18 - 25 July (2004), in Paris, France. p.3954.

7)Bagenal, F., Dowling, T.E., McKinnon, W.B.: Jupiter: The Planet, Satellites and Magnetosphere. Cambridge Univ. Press, 2004

8)Grasset, O. et al.: Satellites of the Outer Solar System, Springer, 2010.

9)McCord, T. B. et al.: Salts an Europa's Surface Detected by Galileo's Near Infrared Mapping Spectrometer. Nature, 280 (1998), pp.1242-1245.

10)Kargel, J. S., et al.: Europa's Crust and Ocean: Origin, Composition, and the Prospects for Life. Icarus, 148 (2000), pp.226-265.

11)Kivelson, M. G., et al.: Discovery of Ganymede's magnetic field by the Galileo spacecraft. Nature, 384 (1996), pp.537-541.

12)Anderson, J. D., et al.: Gravitational Constraints on the Internal Structure of Ganymede. Nature, 384 (1996), pp.541-543.

13)Kimura, J., et al.: Size and Compositional Constraints of Ganymede's Metallic Core for Driving an Active Dynamo. Icarus, 202 (2009), pp.216-224.

14)Morbidelli, M., Levison, H. F., Tsiganis, K. and Gomes, R.: Chaotic Capture of Jupiter's Trojan Asteroids in the Early Solar System, Nature, 435 (2005), pp.462-465.

15)Barucci, M. A. Cruikshank, D. P., Mottola, S., and Lazzarin. M.: Physical Properties of Trojan and Centaur Asteroids, in Asteroids III, Bottke, W. F. et al. (eds.), University of Arizona Press, 2003, pp.273-287.

16)Yano, H. et al.: The Arrayed Large-Area Dust Detectors in Interplanetary Space (ALADDIN) Onboard the IKAROS Solar Sail Demonstrator, 38th COSPAR Scientific Assembly, Bremen (2010) B04-0045-10.s 